\title{
Elliptic Cylinder with Slotted Antenna Coated with Magnetic Metamaterials
}

\author{
A. K. Hamid \\ Electrical and Computer Engineering Department, University of Sharjah, P.O. Box 27272, Sharjah, United Arab Emirates \\ Correspondence should be addressed to A. K. Hamid, akhamid@sharjah.ac.ae \\ Received 20 February 2011; Accepted 16 April 2011 \\ Academic Editor: Matteo Pastorino \\ Copyright ( 2011 A. K. Hamid. This is an open access article distributed under the Creative Commons Attribution License, which \\ permits unrestricted use, distribution, and reproduction in any medium, provided the original work is properly cited. \\ The radiation properties of an axially slotted elliptic antenna coated with magnetic metamaterials are investigated. The fields \\ inside and outside the dielectric coating are expressed in terms of the Mathieu functions. The boundary conditions at various \\ surfaces are enforced using the orthogonality property of angular Mathieu functions. Numerical results are presented graphically \\ for the radiation pattern, aperture conductance, and antenna gain for the TM case. It was found that a slotted antenna coated \\ with magnetic metamaterials has more gain as well as lower side lobes compared to one coated with conventional dielectric or \\ nonmagnetic metamaterials.
}

\section{Introduction}

Radiation properties of an axially slotted antenna are very important in communications and airplane industries. Numerous authors in the literature have investigated the radiation by dielectric-coated slotted circular and elliptical cylinders. For example, Hurd [1] studied the radiation pattern of a dielectric axially slotted cylinder. The external admittance of an axial slot on a dielectric-coated metal cylinder was investigated by Knop [2]. Shafai [3] obtained the radiation properties of an axially slotted antenna coated with a homogenous material. Wong $[4,5]$ investigated the radiation properties of slotted cylinder of elliptical cross-section while Richmond [6] studied the radiation from an axial slot antenna on a dielectric-coated elliptic cylinder. The analysis was later extended to the radiation by axial slots on a dielectric coated nonconfocal conducting elliptic cylinder by Ragheb et al. [7]. Hussein and Hamid [8] studied the radiation by $N$ axially slotted cylinders of elliptical cross section coated with a lossy dielectric material. Recently, A. K. Hamid investigated the radiation characteristics of slotted circular or elliptical cylinder coated with lossy and lossless metamaterials $[9,10]$.

Lately, materials possessing both lossy and lossless metamaterials as well as chiral media have gained considerable attention in many researches [11-24].
In this paper, a theoretical analysis based on a boundary value solution for the case of antenna radiation by an axial slot on a conducting elliptic cylinder coated with magnetic metamaterials is presented. The fields inside and outside the dielectric coating are expressed in terms of radial and angular Mathieu functions. Numerical results are presented for the radiation pattern, aperture conductance, and antenna gain versus coating thickness as well as compared with conventionally dielectric-coated, magnetic and nonmagnetic metamaterial-coated antenna.

\section{Problem Formulation}

The geometry of the perfectly conducting elliptic cylinder with an axially slotted antenna covered with dielectric material is shown in Figure 1.

The structure is assumed to be infinite along the $z$ axis. The symbols $a_{c}$ and $b_{c}$ correspond to the conducting core semimajor and semiminor axes, respectively, while $a$ and $b$ are the semimajor and semiminor axes of the dielectric coating material. The axial slot coordinates on the conducting elliptic cylinder are denoted by $\nu_{1}$ and $\nu_{2}$. The elliptical coordinate system $(u, v, z)$ is assumed, and it can 


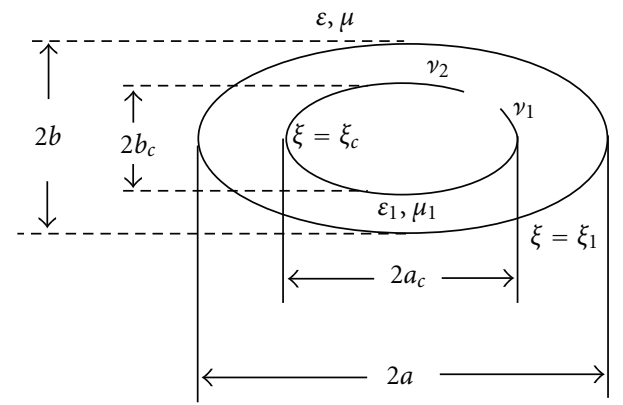

FIGURE 1: Geometry of an axially slotted antenna on elliptic cylinder coated with dielectric material.

be represented in terms of the Cartesian coordinate system $(x, y, z)$ as

$$
\begin{gathered}
x=F \cosh (u) \cos (v), \\
y=F \sinh (u) \sin (v) .
\end{gathered}
$$

$F$ is the semifocal length of the elliptical cross-section. The radiated electric field outside the dielectric coating (region I and $\xi>\xi_{1}$ ) can be expressed in terms of the Mathieu functions as follows:

$$
\begin{aligned}
E_{z}^{\mathrm{I}}= & \sum_{m=0}^{\infty} C_{e m} R_{e m}^{(4)}\left(c_{0}, \xi\right) S_{e m}\left(c_{0}, \eta\right) \\
& +\sum_{m=1}^{\infty} C_{o m} R_{o m}^{(4)}\left(c_{0}, \xi\right) S_{o m}\left(c_{0}, \eta\right),
\end{aligned}
$$

where $C_{e m}$ and $C_{o m}$ are the unknown field expansion coefficients, $S_{e m}$ and $S_{o m}$ are the even odd angular Mathieu functions of order $m$, and $R_{e m}^{(4)}$ and $R_{o m}^{(4)}$ are the even and odd modified Mathieu functions of the fourth kind. It should be noted that $\xi=\cosh u, \eta=\cos v, c_{0}=k F$, and $k=$ $\omega \sqrt{\mu \varepsilon}$. Similarly, the electric field inside the dielectric coating (region II) for $\xi_{c}<\xi<\xi_{1}$ can be expressed in terms of complex Mathieu functions as

$$
\begin{aligned}
E_{z}^{\mathrm{II}}= & \sum_{m=0}^{\infty}\left[A_{e m} R_{e m}^{(1)}\left(c_{1}, \xi\right)+B_{e m} R_{e m}^{(2)}\left(c_{1}, \xi\right)\right] S_{e m}\left(c_{1}, \eta\right) \\
& +\sum_{m=1}^{\infty}\left[A_{o m} R_{o m}^{(1)}\left(c_{1}, \xi\right)+B_{o m} R_{o m}^{(2)}\left(c_{1}, \xi\right)\right] S_{o m}\left(c_{1}, \eta\right),
\end{aligned}
$$

where $c_{1}=k_{1} F, k_{1}=\omega \sqrt{\mu_{1} \varepsilon_{1}}, \underset{\mathrm{om}}{A \text { em }}$ and $\underset{\mathrm{om}}{B \mathrm{em}}$ are the unknown field expansion coefficients, and $R_{\text {om }}^{(1)}$ and $R_{\text {em }}^{(2)}$ are the radial Mathieu functions of the first and second kind, respectively. The angular and the radial Mathieu functions are defined in [15]. The magnetic field component in regions
(I) and (II) is obtained using Maxwell's equations and written as

$$
\begin{aligned}
H_{v}^{\mathrm{I}}= & \frac{-j}{\omega \mu h}\left\{\sum_{m=0}^{\infty} C_{e m} R_{e m}^{(4)^{\prime}}\left(c_{0}, \xi\right) S_{e m}\left(c_{0}, \eta\right)\right. \\
& \left.\quad+\sum_{m=1}^{\infty} C_{o m} R_{o m}^{(4)^{\prime}}\left(c_{0}, \xi\right) S_{o m}\left(c_{0}, \eta\right)\right\}, \\
H_{v}^{\mathrm{II}}= & \frac{-j}{\omega \mu_{1} h} \\
& \times\left\{\sum_{m=0}^{\infty}\left[A_{e m} R_{e m}^{(1)^{\prime}}\left(c_{1}, \xi\right)+B_{e m} R_{e m}^{(2)^{\prime}}\left(c_{1}, \xi\right)\right] S_{e m}\left(c_{1}, \eta\right)\right. \\
& \left.+\sum_{m=1}^{\infty}\left[A_{o m} R_{o m}^{(1)^{\prime}}\left(c_{1}, \xi\right)+B_{o m} R_{o m}^{(2)^{\prime}}\left(c_{1}, \xi\right)\right] S_{o m}\left(c_{1}, \eta\right)\right\},
\end{aligned}
$$

where $h=F \sqrt{\left(\cosh ^{2} u-\cos ^{2} v\right)}$. The prime in (4) denotes the derivative with respect to $u$ while $\mu$ and $\mu_{1}$ are the permeabilities of regions 1 and 2, respectively, and assumed to be purely real numbers.

We require $E_{z}$ to be continuous $\left(E_{z}^{\mathrm{I}}=E_{z}^{\mathrm{II}}\right)$ a cross the interface at $\xi=\xi_{1}$. Applying the orthogonality property of the angular Mathieu functions [16], leads to

$$
\begin{gathered}
{\left[A_{e n} R_{e n}^{(1)}\left(c_{1}, \xi_{1}\right)+B_{e n} R_{e n}^{(2)}\left(c_{1}, \xi_{1}\right)\right] N_{e n}\left(c_{1}\right)} \\
=\sum_{m=0}^{\infty} C_{e m} R_{e m}^{(4)}\left(c_{0}, \xi_{1}\right) M_{e n m}\left(c_{1}, c_{0}\right),
\end{gathered}
$$

where

$$
\begin{gathered}
N_{e n}\left(c_{1}\right)=\int_{0}^{2 \pi}\left[S_{e n}\left(c_{1}, \eta\right)\right]^{2} d v \\
M_{e n m}\left(c_{1}, c_{0}\right)=\int_{0}^{2 \pi} S_{e n}\left(c_{1}, \eta\right) S_{e m}\left(c_{0}, \eta\right) d v .
\end{gathered}
$$

Continuity of the tangential magnetic field components at $\xi=\xi_{1}$ requires that

$$
\begin{array}{r}
{\left[A_{e n} R_{e n}^{(1)^{\prime}}\left(c_{1}, \xi_{1}\right)+B_{e n} R_{e n}^{(2)^{\prime}}\left(c_{1}, \xi_{1}\right)\right] N_{e n}\left(c_{1}\right)} \\
=\frac{\mu_{1}}{\mu} \sum_{m=0}^{\infty} C_{e m} R_{e m}^{(4)^{\prime}}\left(c_{0}, \xi_{1}\right) M_{e n m}\left(c_{1}, c_{0}\right) .
\end{array}
$$

Similar equations for the odd solution are needed and may be obtained by replacing " $e$ " with " $o$ " in (5) and (7). In region 
(II), the tangential electric field on the conducting surface $\left(\xi=\xi_{c}\right)$ must vanish except at the slot location. This leads to

$$
\begin{aligned}
\sum_{m=0}^{\infty}[ & \left.A_{e m} R_{e m}^{(1)}\left(c_{1}, \xi_{c}\right)+B_{e m} R_{e m}^{(2)}\left(c_{1}, \xi_{c}\right)\right] S_{e m}\left(c_{1}, \eta\right) \\
& +\sum_{m=1}^{\infty}\left[A_{o m} R_{o m}^{(1)}\left(c_{1}, \xi_{c}\right)+B_{o m} R_{o m}^{(2)}\left(c_{1}, \xi_{c}\right)\right] S_{o m}\left(c_{1}, \eta\right) \\
= & \begin{cases}F(v), & v_{1}<v<v_{2}, \\
0, & \text { elsewhere. }\end{cases}
\end{aligned}
$$

$F(v)$ represents the unknown aperture fields on the slot which are expressed in terms of sinusoidal Fourier representation of even and odd parts. The field at the slot location may be expressed as

$$
\begin{gathered}
F(v)=E_{0} \cos \left[\frac{\pi\left(\nu_{0}-\nu\right)}{2 \alpha}\right], \\
\nu_{0}=\frac{\left(\nu_{1}+\nu_{2}\right)}{2}, \\
\alpha=\frac{\left(\nu_{2}-\nu_{1}\right)}{2} .
\end{gathered}
$$

Multiplying both sides of (8) by $S_{e n}\left(c_{1}, \eta\right)$ and integrating over $0<v<2 \pi$, we obtain

$$
\begin{gathered}
{\left[A_{e n} R_{e n}^{(1)}\left(c_{1}, \xi_{c}\right)+B_{e n} R_{e n}^{(2)}\left(c_{1}, \xi_{c}\right)\right] N_{e n}\left(c_{1}\right)} \\
\quad=F_{e n} \\
\quad=\int_{v_{1}}^{\nu_{2}} F(v) S_{e n}\left(c_{1}, \eta\right) d v .
\end{gathered}
$$

Solving for $B_{e n}$ from (10) and using the result in (5) and (7) with the elimination of $A_{e n}$, we obtain a system of linear equations in terms of $\mathrm{C}_{\mathrm{en}}$.

\section{Numerical Results}

Once the unknown field expansion coefficients $\underset{\substack{e n \\ o n}}{C}$ are computed, quantities of interest such as far-field radiation pattern, antenna gain, and the aperture conductance can be obtained. The far-zone radiation pattern for the electric field can be calculated using the asymptotic form of the radial Mathieu functions $R_{e m}^{(4)}$. Thus the far-zone field of the slot antenna can be written as

$$
\begin{aligned}
& E_{z}^{\mathrm{I}}(\rho, \varphi) \\
& =\sqrt{\frac{j}{k \rho}} e^{-j k \rho} \\
& \quad \times\left[\sum_{n=0}^{\infty} j^{n} C_{e n} S_{e n}\left(c_{0}, \cos \varphi\right)+\sum_{n=1}^{\infty} j^{n} C_{o n} S_{o n}\left(c_{0}, \cos \varphi\right)\right],
\end{aligned}
$$

where $\rho$ and $\varphi$ denote the polar coordinates in the circular cylindrical system. The antenna gain is expressed as [6-10]

$$
\begin{aligned}
G(\varphi)=\frac{1}{Z_{0} k \rho}\left[\left|\sum_{n=0}^{\infty} j^{n} C_{e n} S_{e n}\left(c_{0}, \cos \varphi\right)\right|^{2}\right. \\
\left.+\left|\sum_{n=1}^{\infty} j^{n} C_{o n} S_{o n}\left(c_{0}, \cos \varphi\right)\right|^{2}\right],
\end{aligned}
$$

where $Z_{0}$ is the free space impedance. The aperture conductance per unit length of the slot antenna is defined as [6]

$$
G_{a}=2 \pi \rho \frac{S_{\mathrm{av}}}{\left|E_{0}\right|^{2}}
$$

where $S_{\mathrm{av}}$ is the average power density averaged over an imaginary cylinder of radius $\rho$ and given as

$$
S_{\mathrm{av}}=\frac{1}{2 \pi Z_{0} k \rho}\left[\sum_{n=0}^{\infty}\left|C_{e n}\right|^{2} N_{e n}\left(c_{0}\right)+\sum_{n=1}^{\infty}\left|C_{o n}\right|^{2} N_{o n}\left(c_{0}\right)\right] .
$$

The accuracy of our numerical results is verified against published results for a single slotted circular or elliptic antenna coated with a lossless conventional dielectric material [6]. The geometrical parameters of the slotted antenna used for comparison are $a_{c}=\lambda, b_{c}=\lambda / 2, b=b_{c}+t$, where $t$ is the coating thickness, $\nu_{0}=90^{\circ}$ and $\alpha=2.8657^{\circ}$. Figure 2 shows the radiation pattern numerical results (gain versus $\varphi$ ) obtained for a conventional dielectric coating material represented by solid line, for comparison [6] $\left(\varepsilon_{r}=4\right.$ and $\left.\mu_{r}=1\right)$, nonmagnetic metamaterials coating represented by dotted line $\left(\varepsilon_{r}=-4\right.$ and $\left.\mu_{r}=-1\right)$, and magnetic metamaterial coating represented by circles $\left(\varepsilon_{r}=-4\right.$ and $\mu_{r}=$ $-1.5)$. It can be seen that the magnetic metamaterials coating material reduces the side-lobes more than the nonmagnetic case. The effect of the coating thickness is illustrated in Figure 3 for the same geometrical parameters as in Figure 2. One may notice that by increasing the thickness of the magnetic metamaterials, coating enhances the gain with a decrease in the number of side lobes. On the other hand, it was earlier shown in $[6,8]$ that by increasing the thickness of the conventional dielectric, coating $\left(\varepsilon_{r}=4\right.$ and $\left.\mu_{r}=1\right)$ results in a reduction of the main beam with an increase in the number of side lobes.

The gain versus coating thickness for a slotted elliptical antenna with the same geometrical parameters used in Figure 3 is displayed in Figure 4 . The gain is evaluated at $\varphi=90^{\circ}$ since the slot is centered at $\nu=90^{\circ}$ where the gain is expected to be maximum.

The gain versus coating layer material permeability is plotted in Figure 5. When $t=0.15 \lambda$, the gain has almost constant gain $-4 \leq \mu_{r} \leq-1$ and the gain oscillates when $t=0.3 \lambda$. 


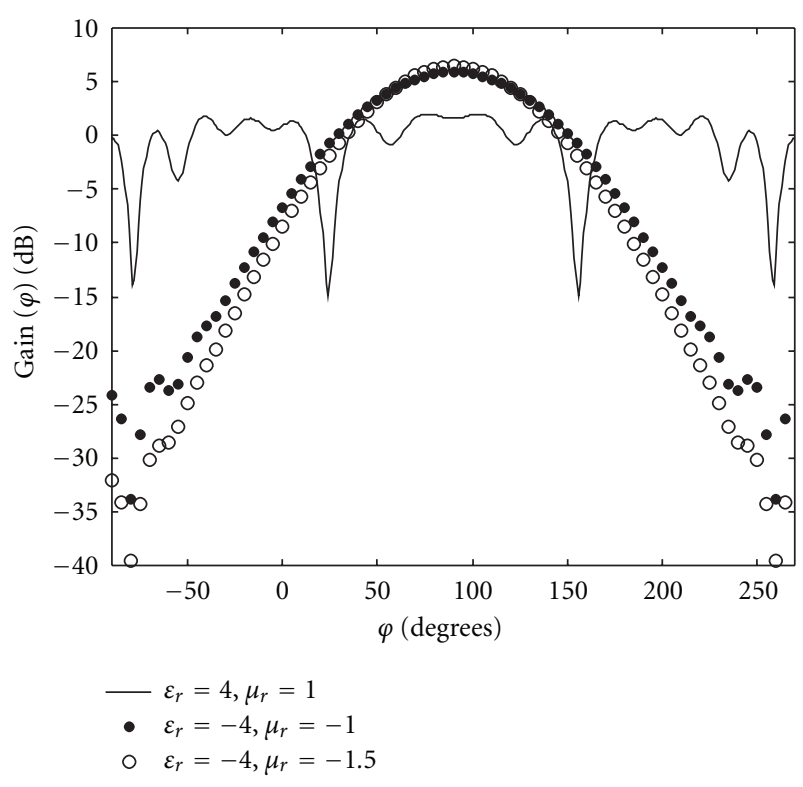

FIGURE 2: Radiation pattern of an axially slotted elliptic cylinder coated with conventional dielectric, magnetic and nonmagnetic metamaterials. $a_{c}=\lambda, b_{c}=\lambda, a=b=b_{c}+0.25 \lambda, v_{0}=90^{\circ}$, and $\alpha=2.8657^{\circ}$.

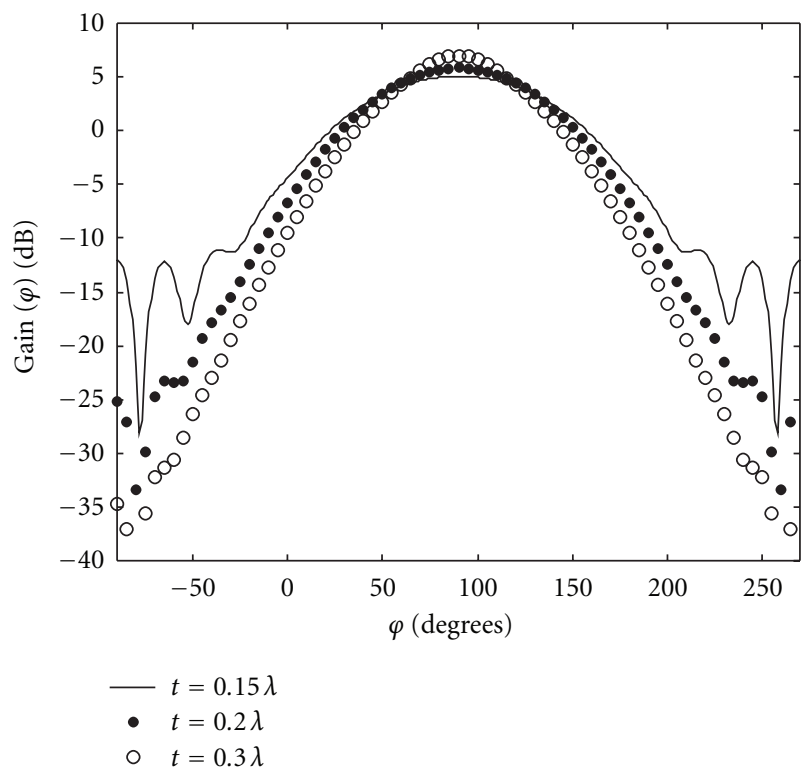

FIGURE 3: Radiation pattern of an axially slotted elliptic cylinder coated with magnetic metamaterials. $a_{c}=\lambda, b_{c}=\lambda / 2, b=b_{c}+t$, $v_{0}=90^{\circ}, \alpha=2.8657^{\circ}, \varepsilon_{r}=-4.0$, and $\mu_{r}=-1.5$.

\section{Conclusions}

The radiation characteristics of an axially slotted elliptic antenna coated with magnetic metamaterials were investigated using analytic solution. It was also shown that the presence of magnetic metamaterials coating has changed significantly the characteristics of the antenna. Finally, the

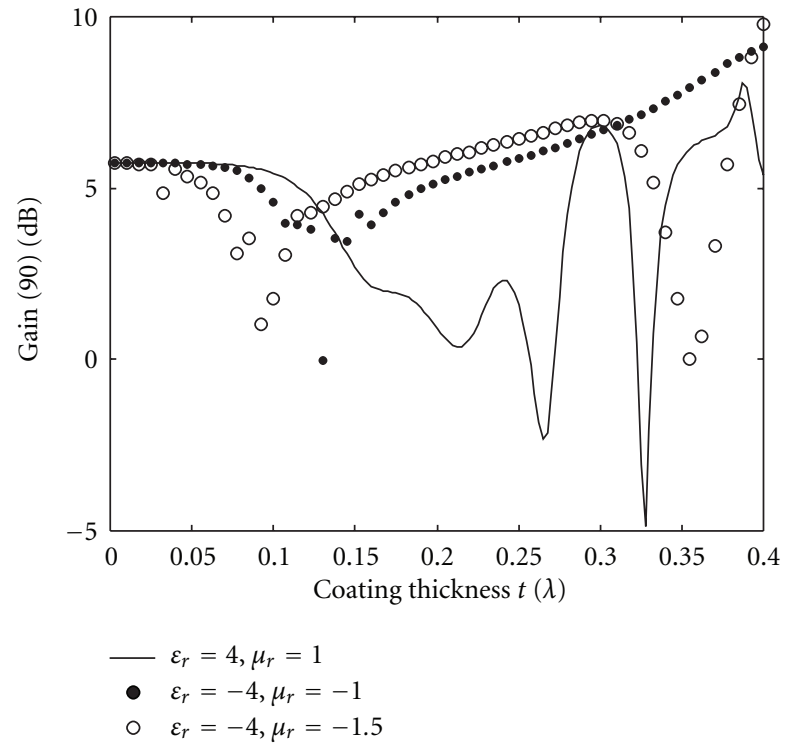

Figure 4: Gain versus coating thickness for an axially slotted elliptic cylinder coated with conventional dielectric, magnetic and nonmagnetic metamaterials. $a_{c}=\lambda, b_{c}=\lambda / 2, b=b_{c}+t, v_{0}=90^{\circ}$, and $\alpha=2.8657^{\circ}$.

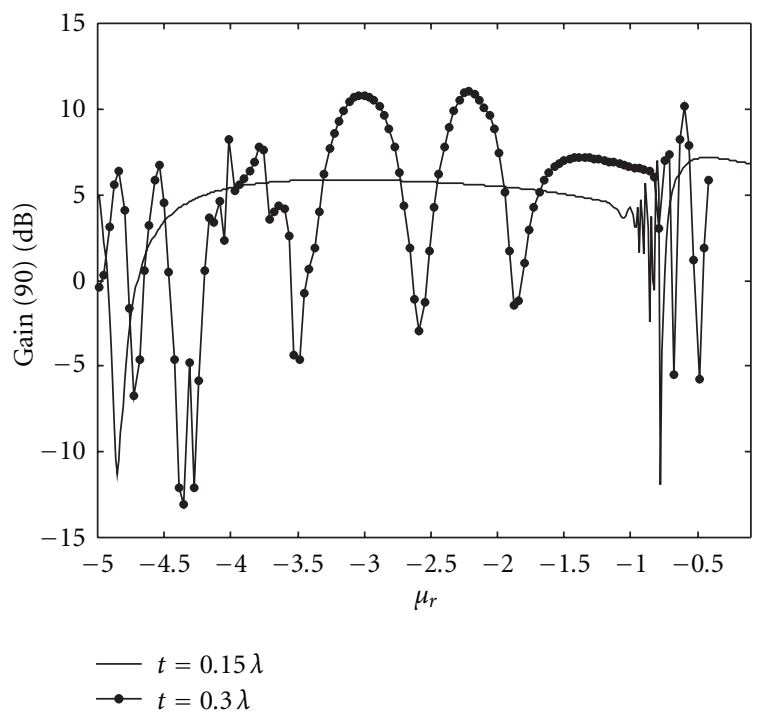

FIGURE 5: Gain versus $\mu_{r}$ for an axially slotted elliptic cylinder coated with magnetic metamaterials. $a_{c}=\lambda, b_{c}=\lambda / 2, b=b_{c}+t$, $\nu_{0}=90^{\circ}, \alpha=2.8657^{\circ}$, and $\varepsilon_{r}=-4.0$.

magnetic metamaterials can be used to enhance the antenna gain and lower the side lobes by using some specific values of magnetic coating metamaterials.

\section{Acknowledgment}

The author wishes to acknowledge the support provided by the University of Sharjah, UAE. 


\section{References}

[1] R. A. Hurd, "Radiation patterns of a dielectric coated axially slotted cylinder," Canadian Journal of Physics, vol. 34, pp. 638642, 1956.

[2] C. M. Knop, "External admittance of an axial slot on a dielectric coated metal cylinder," Radio Science, vol. 3, pp. 803$818,1968$.

[3] L. Shafai, "Radiation from an axial slot antenna coated with a homogeneous material," Canadian Journal of Physics, vol. 50, no. 23, pp. 3072-3077, 1972.

[4] J. Y. Wong, "Radiation pattern of slotted elliptic cylinder antenna," IEEE Transactions on Antennas and Propagation, vol. 3, pp. 200-203, 1955.

[5] J. Y. Wong, "Radiation conductance of axial and transverse slots in cylinders of elliptical cross section," Proceedings of the IRE, vol. 41, pp. 1172-1177, 1953.

[6] J. H. Richmond, "Axial slot antenna on dielectric-coated elliptic cylinder," IEEE Transactions on Antennas and Propagation, vol. 37, no. 10, pp. 1235-1241, 1989.

[7] H. A. Ragheb, A. Sebak, and L. Shafi, "Radiation by axial slots on a dielectric-coated nonconfocal conducting elliptic cylinder," IEE Proceedings: Microwaves, Antennas and Propagation, vol. 143, no. 2, pp. 124-130, 1996.

[8] M. I. Hussein and A. K. Hamid, "Radiation characteristics of N axial slots on a conducting elliptical antenna coated by a lossy dielectric layer," Canadian Journal of Physics, vol. 82, no. 2, pp. 141-149, 2004.

[9] A. K. Hamid, "Axially slotted antenna on a circular or elliptic cylinder coated with metamaterials," Progress in Electromagnetic Research, vol. 51, pp. 329-341, 2005.

[10] A. K. Hamid, "Study of lossy effects on the characteristics of axially slotted circular or elliptical cylindrical antennas coated with metamaterials," IEE Proceedings: Microwaves, Antennas and Propagation, vol. 152, no. 6, pp. 485-490, 2005.

[11] R. Ruppin, "Extinction properties of a sphere with negative permittivity and permeability," Solid State Communications, vol. 116, no. 8, pp. 411-415, 2000.

[12] S. A. Tretyakov, S. I. Maslovski, A. A. Sochava, and C. R. Simovski, "The influence of complex material coverings on the quality factor of simple radiating systems," IEEE Transactions on Antennas and Propagation, vol. 53, no. 3, pp. 965-970, 2005.

[13] N. Engheta and R. W. Ziolkowski, "A positive future for double-negative metamaterials," IEEE Transactions on Microwave Theory and Techniques, vol. 53, no. 4, pp. 15351555, 2005.

[14] E. Shamonina and L. Solymar, "Properties of magnetically coupled metamaterial elements," Journal of Magnetism and Magnetic Materials, vol. 300, no. 1, pp. 38-43, 2006.

[15] A. K. Hamid, "Multi-dielectric loaded axially slotted antenna on circular or elliptic cylinder," Journal of Electromagnetic Waves and Applications, vol. 20, no. 9, pp. 1259-1271, 2006.

[16] B. N. Khatir, M. Al-Kanhal, and A. Sebak, "Electromagnetic wave scattering by elliptic chiral cylinder," Journal of Electromagnetic Waves and Applications, vol. 20, no. 10, pp. 13771390, 2006.

[17] B. N. Khatir and A. R. Sebak, "Slot antenna on a conducting elliptic cylinder coated by chiral media," Electromagnetics, vol. 29, no. 7, pp. 522-540, 2009.

[18] B. N. Khatir and A. R. Sebak, "Slot antenna on a conducting elliptic cylinder coated by nonconfocal chiral media," Progress in Electromagnetics Research, vol. 93, pp. 125-143, 2009.

[19] M. Pastorino, Microwave Imaging, Wiley, Hoboken, NJ, USA, 2010.
[20] N. B. Kakogiannos and J. A. Roumeliotis, "Electromagnetic scattering from an infinite elliptic metallic cylinder coated by a circular dielectric one," IEEE Transactions on Microwave Theory and Techniques, vol. 38, no. 11, pp. 1660-1666, 1990.

[21] S. Caorsi, M. Pastorino, and M. Raffetto, "Scattering by a conducting elliptic cylinder with a multilayer dielectric coating," Radio Science, vol. 32, no. 6, pp. 2155-2166, 1997.

[22] S. Caorsi and M. Pastorino, "Scattering by multilayer isorefractive elliptic cylinders," IEEE Transactions on Antennas and Propagation, vol. 52, no. 1, pp. 189-196, 2004.

[23] S. Caorsi, M. Pastorino, and M. Raffetto, "Electromagnetic scattering by weakly lossy multilayer elliptic cylinders," IEEE Transactions on Antennas and Propagation, vol. 46, no. 11, pp. 1750-1751, 1998.

[24] M. Pastorino, M. Raffetto, and A. Randazzo, "Interactions between electromagnetic waves and elliptically shaped metamaterials," IEEE Antennas and Wireless Propagation Letters, vol. 4, no. 1, pp. 165-168, 2005. 

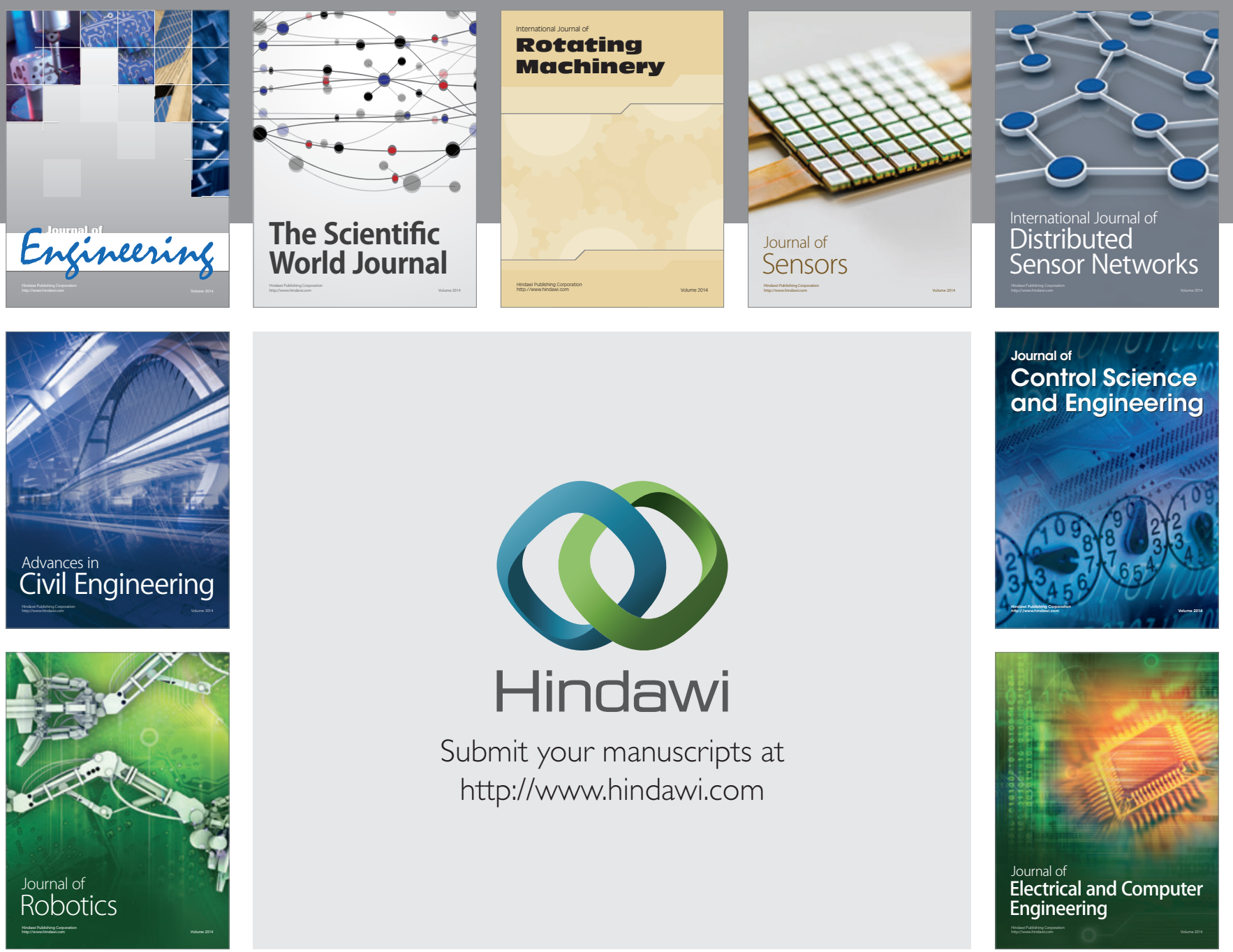

Submit your manuscripts at

http://www.hindawi.com
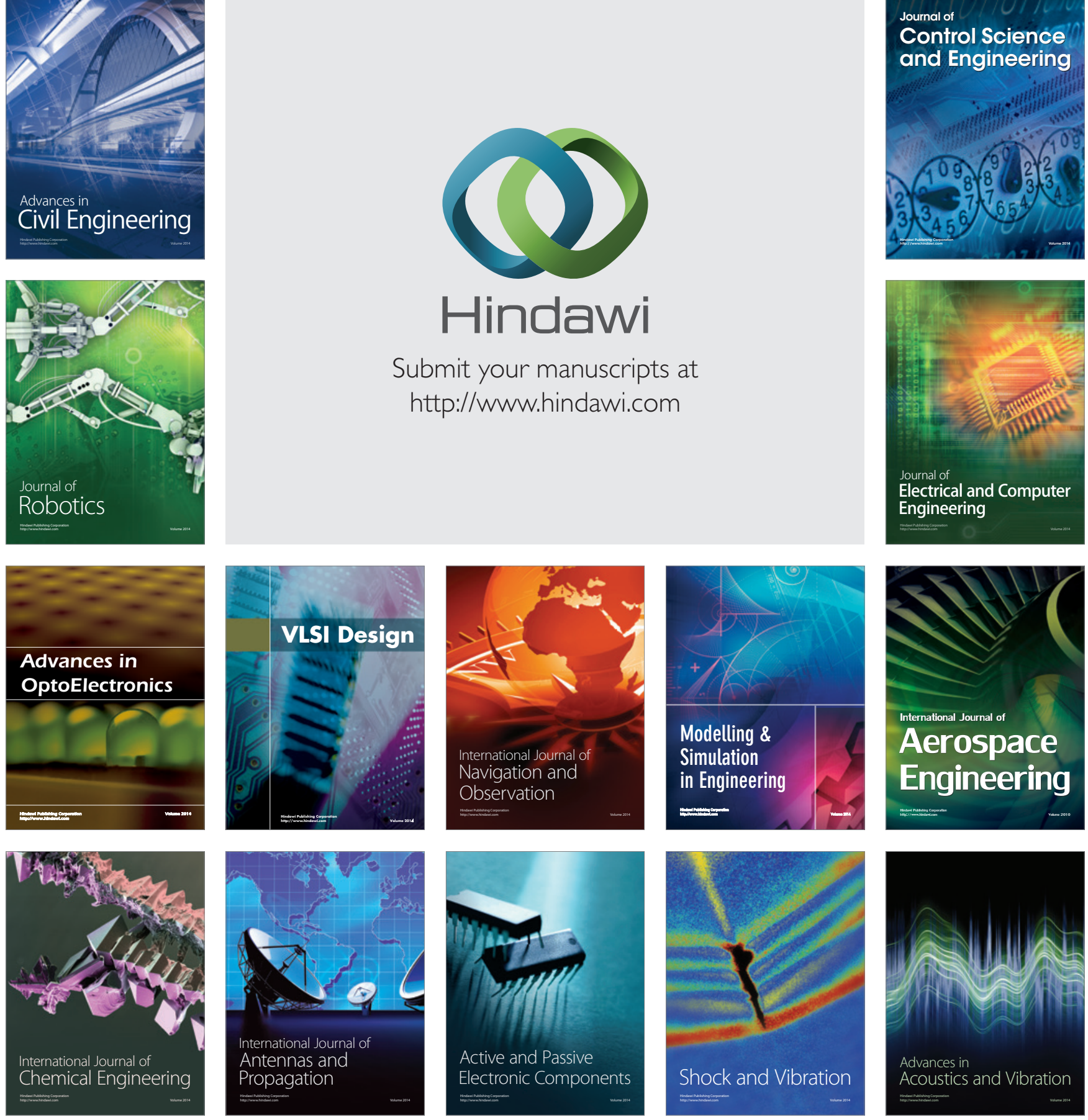